\title{
REM sleep and the timing of self-awakenings
}

\author{
HAROLD ZEPELIN \\ Oakland University, Rochester, Michigan
}

\begin{abstract}
For insight into the reputed accuracy of some people's time judgment during sleep, differences between sleep stages in the threshold for self-awakening were investigated. Self-awakenings by 11 sleepers indicated that the threshold is lowest during REM sleep. The cyclical recurrence of REM sleep apparently acts as an interval-timing clock which motivated sleepers can use to some advantage, but the inconsistent performance of occasionally accurate awakeners casts doubt on the existence of any time-telling ability.
\end{abstract}

A lingering puzzle with a long history is the reputed ability of some people to awaken themselves punctually at predetermined, nonhabitual times without benefit of external cues. Coming mainly from studies in which sleepers acted as their own observers (Clauser, 1954; Frobenius, 1927; Vaschide, 1911), reports of such accurate time judgment prompt skeptical appraisal. A little-noted deficiency common to these reports is their failure to distinguish between truly accurate single awakenings and what amounts to inaccurate performance when a sleeper awakes prematurely, perhaps consults a clock, and then manages to awake near a target time on a second or third attempt. More credible accounts have come from contemporary investigations with continuous electrophysiological sleep recordings (Lavie, Oksenberg, \& Zomer, 1979; Zung \& Wilson, 1971). However, even the relatively modest claims of accuracy in these studies leave ground for disbelief.

Causing doubt is the fact that Zung and Wilson's (1971) sleepers, who were randomly chosen (i.e., not selected for self-awakening ability), performed with as much accuracy as those studied by Lavie et al. (1979), who were confident of their ability because of reportedly successful prior experience. Moreover, sleepers known to have awakened accurately on one or two occasions have been strikingly inconsistent, doing poorly when called upon for repeat performance with varied target times (Lavie et al., 1979; Tart, 1970). Furthermore, statistical tests of accuracy have been based on the assumption that, by chance, there is an equal probability of self-awakening at all times during sleep (Tart, 1970; Zung \& Wilson, 1971). This assumption is gratuitous. The threshold for self-awakening could vary with sleep stage, as does the threshold for awakening to auditory stimuli. There also is evidence that the electroencephalographic (EEG) stages differ with respect to recall and execution of presleep instructions (e.g., to press a microswitch in response to a signal), with superior performance during rapid eye movement (REM) sleep (Williams, Morlock, \& Morlock, 1966). A reason-

This research was conducted in the laboratory of Georges R. Reding at the University of Chicago. The author's mailing address is: Department of Psychology, Oakland University, Rochester, MI 48063. able hypothesis is that the threshold for self-awakening is lowest during REM sleep, as Lavie et al. (1979) suggested. This possibility was examined in a previously unpublished exploratory study (Zepelin, 1967, 1968).

\section{METHOD}

Prospective subjects were screened for soundness of sleep in order to minimize the possibility of adventitious awakenings that could distort the experimental data. Those selected were 7 men and 8 women (ages 15-32 years), whose responses on sleep questionnaires indicated that they ordinarily rarely awoke at night. Following a laboratoryadaptation night, 12 of these subjects slept with EEG and electrooculographic (EOG) recordings on a nonconsecutive screening night for $5 \mathrm{~h}$ or more before any spontaneous awakening. (The 5-h period exceeded the assigned sleep durations on experimental nights.) The other 3 subjects each awoke once within the 5-h period but were included, since sleepers who could meet the 5-h criterion proved to be rare. Screening-night records provided a baseline of each subject's sleep patterns. Also available as baseline data for all subjects were two or three records obtained on nights devoted to other research that involved no interference with sleep.

Four subjects described themselves as accurate self-awakeners; the other 11 were simply "willing to try." All were paid a nightly fee, with bonuses related to accuracy of self-awakening (SA). For SA trials, subjects slept on nonconsecutive laboratory nights with continuous EEG and EOG recordings in sound-attenuated bedrooms that were completely shielded from outside light. The experimenter monitored recordings in an adjacent room, with an intercom in constant operation for communication with the sleepers, who were instructed to call out whenever they awoke. Any awakenings related to transient disturbances (e.g., thunder) were readily recognizable. Otherwise, every awakening was treated as an intentional act based on the sleeper's effort to judge time.

Sleepers were informed of the correct time when bedroom lights were turned out. Target times were near the middle of the night, when sleep stages tend toward equal representation. Target time was 0400 on 5 nights, with assigned preawakening intervals ranging from 198 to 266 min because of varied bedtimes. For the remaining trials, target time was 0300 with bedtimes at or near 2330 , so the assigned preawakening intervals ranged from 207 to $210 \mathrm{~min}$.

\section{RESULTS}

Eleven of the 15 sleepers managed to awake. Evaluation of stage differences was based on these SAs. In some instances, when SAs were considerably premature, additional attempts to awake at target-time were allowed. These played no part in the evaluation of stage differences, but provided supplementary information. Sleep stages 
were scored in accordance with the criteria commonly employed at the time of the study (Dement \& Kleitman, 1957).

Of the 11 SAs, 2 were from delta sleep (Stages 3 and 4 combined), 5 were from Stage 2, and 4 were from REM sleep. The probability of SAs from the different stages was estimated from the representation of each stage during the $660 \mathrm{~min}$ of sleep (i.e., $1 \mathrm{~h}$ for each of the 11 awakeners) directly preceding the SAs. These proportions were $31 \%$ for delta sleep, $53 \%$ for Stage 2 , and $9 \%$ for REM sleep. (NonREM Stage 1 occupied 7\%. Since Stage 1 is a transitional stage mainly associated with arousals, SAs occurring in conjunction with it were assigned to the major stages from which the arousals developed.) The percentage of SAs from Stage 2 was in keeping with expectations based on chance, whereas the percentage of SAs from delta sleep fell below expectations. The percentage from REM sleep significantly exceeded expectations. By binomial test, the probability of 4 SAs from REM rather than nonREM sleep was .01.

All but one of the SAs were premature. Absolute errors of timing ranged from 7 to $131.5 \mathrm{~min}$, with a median of $54 \mathrm{~min}$. However, SA frequency increased as sleep accumulated. There were no SAs during the first hour of the sleep period, and only two during the second hour, but five in the third hour and four in the fourth hour. Two SAs were within 10 min of target time, and another was within $15 \mathrm{~min}$, all of these by self-described accurate awakeners. Since these results suggested the possible operation of time judgment, the 2 most accurate awakeners were tested with additional trials. Asked to awake accurately a second time under the same conditions, the most accurate subject awoke $24.5 \mathrm{~min}$ early, and the other awoke 49.5 min early. On a third trial, with target time $1 \mathrm{~h}$ later (preawakening interval $=270 \mathrm{~min}$ ), the most accurate subject awoke 127 min early.

Comparisons of the most accurate awakener's sleep stage patterns on experimental and baseline nights indicated that the timing of his REM periods might explain his accuracy. On 3 baseline nights, his first and second REM periods were separated by 78.5-91.0 min of nonREM sleep. On the baseline night depicted in Figure 1, this interval was $85.5 \mathrm{~min}$. The accurate SA on his first trial, also shown in Figure 1, occurred out of a REM period that began $78.5 \mathrm{~min}$ after the first one. The inaccurate SA on his second trial began in Stage 2, $80.5 \mathrm{~min}$ after the first REM period. Prematurity here was related largely to the shortened first REM period, which lasted only $5 \mathrm{~min}$, instead of the $18 \mathrm{~min}$ on his first trial. The striking correspondence between the timing of these SAs and the subject's interREM intervals raises the possibility that the SA occurred in the course of a transition to REM sleep, or at the very outset of the second REM period. (When such awakening occurs, pinpointing a transition to REM sleep is not possible because the lowvoltage background activity in the Stage 2 EEG that usually precedes REM sleep is similar to the REM EEG.
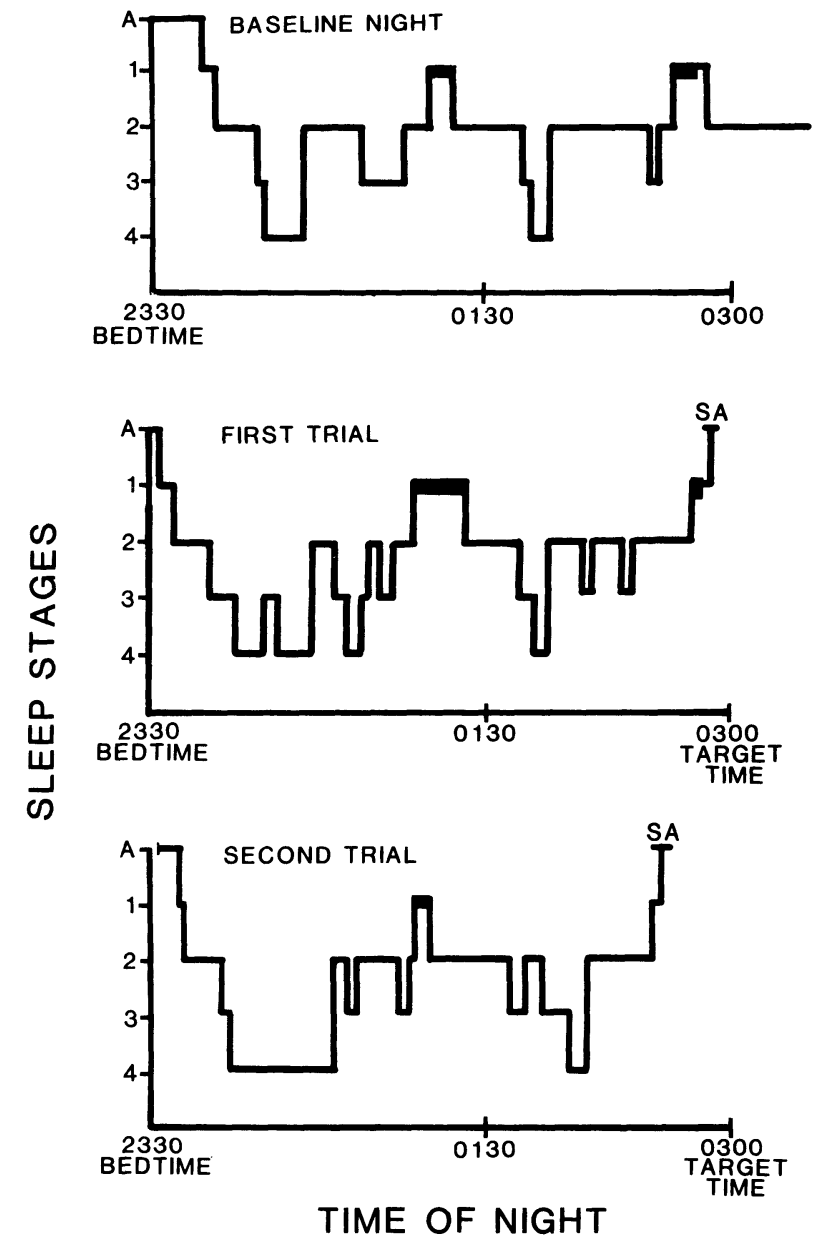

A-AWAKE -REM SLEEP

SA-SELF-AWAKENING

Figure 1. Cycling of sleep stages on the baseline night of the most accurate self-awakener compared with cycling during his most accurate trials.

Eye movements typically appear later.) It is noteworthy that the same sleeper was grossly inaccurate on his third trial because he awoke from Stage 2 only $35.5 \mathrm{~min}$ after his first REM period.

In the 11 trials employed to test stage differences, parallels between the timing of SAs and the duration of interREM intervals characteristic of individual sleepers were found for 3 of the 5 SAs from Stage 2. If, in fact, these SAs occurred at points of transition to REM sleep, it would mean that 7 out of the 11 SAs were REM-related.

Taking into account SAs that occurred when subjects were allowed more than one attempt per night to awake accurately, there were 20 SAs in all, 8 of them clearly from REM, plus the 3 from suspected REM transitions. With repeated awakenings allowed, there were 6 sleepers who managed to awaken within 15 min of target time. This included one inexperienced subject who erred by only half a minute with the second SA of the night. 


\section{DISCUSSION}

The present findings, along with those of Lavie et al. (1979), indicate that the threshold for self-awakening is lowest during REM sleep. Zung and Wilson's (1971) report of no threshold difference between stages is open to question because their assignment of awakenings to nonREM Stage 1 may have obscured a difference between the major stages. In addition to showing stage differences, the present findings indicate that the threshold becomes lower as the night progresses.

An alternative interpretation of the observed distribution of awakenings is that they were prompted or influenced by sleepers' time judgments. The obstacle to this interpretation is the lack of independent evidence for time judgment per se. In the absence of demonstrations that there are individuals who can awake accurately with some consistency at a variety of target times, there is no objective support for the belief in time-telling ability during sleep. Reports of such demonstrations antedating the modern era of sleep research must be discounted for reasons previously given. Contemporary studies have yet to identify a single individual capable of consistent performance.

As noted by Lavie et al (1979) and documented in the present study, accurate awakenings are likely to occur by virtue of temporal proximity between target times and REM periods. REM probably facilitates both the recall of the intention to awake and the act of awakening itself. Its cyclical recurrence seems to provide an interval-timing clock that is available to all who are sufficiently motivated to awake and whose sleep is not so deep as to prevent it. With the opportunity for repeated awakenings during the same night, many awakenings can seem "accurate."

Further investigation of the possible relationship between selfawakenings and transitions to REM sleep would be useful. A shortcoming of the present study was the absence of electromyograms (EMGs), which might have pinpointed REM onsets by showing the associated loss of muscle tone.

\section{REFERENCES}

Clauser, G. (1954). Die Kopfuhr: das automatische Erwachen. Stuttgart: Enke.

Dement, W. C., \& Kleitman, N. (1957). Cyclic variations in EEG during sleep and their relation to eye movements, body motility, and dreaming. Electroencephalography \& Clinical Neurophysiology, 9, 673-690.

FrobenIUS, K. (1927). Über die zeitliche Orientierung im Schlaf und einige Aufwechphänomene. Zeitschrift für Psychologie, 103, 100-110.

LAVIE, P., OKSenberg, A., \& Zomer, J. (1979). It's time, you must wake up now. Perceptual \& Motor Skills, 49, 447-450.

TART, C. T. (1970). Waking from sleep at a preselected time. Journal of American Society of Psychosomatic Dentistry \& Medicine, 17, 3-16.

VASChIDE, N. (1911). Le sommeil et les réves. Paris: Flammarion.

Williams, H. L., Morlock, H. C., \& Morlock, J. V. (1966). Instrumental behavior during sleep. Psychophysiology, 2, 208-216.

ZEPELIN, H. (1967). Self-awakening and the sleep cycle. Psychophysiology, 4, 370 (Abstract).

ZEPELIN, H. (1968). Temporal orientation and self-awakening from sleep. Unpublished master's thesis. Roosevelt University, Chicago.

Zung, W. W. K., \& WILson, W. P. (1971). Time estimation during sleep. Biological Psychiatry, 3, 159-164.

(Manuscript received for publication March 10, 1986.) 